\title{
Toward Silicon-Based Lasers for Terahertz Sources
}

\author{
Stephen A. Lynch, Senior Member, IEEE, Douglas J. Paul, Senior Member, IEEE, \\ Paul Townsend, Student Member, IEEE, Guy Matmon, Student Member, IEEE, Zhang Suet, Student Member, IEEE, \\ Robert W. Kelsall, Zoran Ikonic, Paul Harrison, Senior Member, IEEE, Jing Zhang, David J. Norris, \\ Anthony G. Cullis, Carl R. Pidgeon, Pawel Murzyn, Ben Murdin, Mike Bain, Harry S. Gamble, Member, IEEE, \\ Ming Zhao, and Wei-Xin Ni
}

\begin{abstract}
Producing an electrically pumped silicon-based laser at terahertz frequencies is gaining increased attention these days. This paper reviews the recent advances in the search for a siliconbased terahertz laser. Topics covered include resonant tunneling in p-type $\mathrm{Si} / \mathrm{SiGe}$, terahertz intersubband electroluminescence from quantum cascade structures, intersubband lifetime measurements in $\mathrm{Si} / \mathrm{SiGe}$ quantum wells, enhanced optical guiding using buried silicide layers, and the potential for exploiting common impurity dopants in silicon such as boron and phosphorus to realize a terahertz laser.
\end{abstract}

Index Terms-Boron, far infrared, germanium, impurity, lifetime, phosphorus, pump-probe, quantum cascade laser, resonant tunneling diode (RTD), silicide, silicon, terahertz, waveguide.

\section{INTRODUCTION}

$\mathbf{S}$ EMICONDUCTOR lasers are gaining increasing importance in industrial applications and there have been sustained efforts over the past few years to develop a silicon-based laser source. Such a silicon-based device would represent a considerable advancement in optoelectronic technology, since it would open the way for inexpensive monolithic integrated optical and electronic components.

While III-V semiconductor lasers covering the ultraviolet to the far infrared wavelength ranges are now commonplace, efficient silicon-based lasers have consistently eluded researchers.

Manuscript received October 31, 2005; revised August 24, 2006. This work was supported in part by the EU under Grant SHINE IST-2001-38035, in part by the U.S. DARPA under Air Force Contract F-19628-99-C-0074, and in part by the U.K. EPSRC under Grant GR/S27535/01.

S. A. Lynch, D. J. Paul, P. Townsend, G. Matmon, and Z. Suet are with the Cavendish Laboratory, University of Cambridge, Cambridge CB3 0HE, U.K. (e-mail: lynchs@ieee.org; dp109@cam.ac.uk; pt261@cam.ac.uk; gm331@cam.ac.uk; zys20@cam.ac.uk).

R. W. Kelsall, Z. Ikonic, and P. Harrison are with the School of Electronic and Electrical Engineering, University of Leeds, Leeds LS2 9JT, U.K. (e-mail: r.w.kelsall@leeds.ac.uk; eenzi@ee.leeds.ac.uk; p.harrison@ee.leeds.ac.uk).

J. Zhang is with the Blackett Laboratory, Imperial College, London SW7 2AZ, U.K. (e-mail: jing.zhang@imperial.ac.uk).

D. J. Norris and A. G. Cullis are with the School of Electronics and Electrical Engineering, The University of Sheffield, Sheffield S10 2TN, U.K. (e-mail: d.j.norris@sheffield.ac.uk; a.g.cullis@sheffield.ac.uk).

C. R. Pidgeon and P. Murzyn are with the Department of Physics, HeriotWatt University, Edinburgh EH14 4AS, U.K. (e-mail: c.r.pidgeon@hw.ac.uk; p.murzyn@hw.ac.uk).

B. Murdin is with the School of Electronics and Physical Sciences, University of Surrey, Surrey GU2 7XH, U.K.

M. Bain and H. S. Gamble are with the School of Electrical and Electronic Engineering, Queens University, Belfast BT9 5AH, U.K. (e-mail: m.bain@ee.qub.ac.uk; h.gamble@ee.qub.ac.uk).

M. Zhao and W.-X. Ni are with the Department of Physics, Linköping University, SE-58183 Linköping, Sweden (e-mail: minzh@ifm.liu.se; wxn@ifm. liu.se).

Digital Object Identifier 10.1109/JSTQE.2006.884069
This is largely due to the indirect bandgap in silicon, which prevents fast electron-hole recombination. Quantum cascade lasers, however, rely on intersubband transitions, effectively negating effects resulting from the detrimental indirect bandgap [1]. Intersubband transitions in $\mathrm{Si} / \mathrm{SiGe}$ heterostructures have small energy gaps, resulting in emission in the mid- and far-infrared (terahertz) part of the electromagnetic spectrum [2]-[4]. Recently, there has also been considerable interest in using impurity dopants in silicon such as boron and phosphorus, since it was demonstrated that such materials can show electroluminescent emission in the terahertz region of the electromagnetic spectrum [5]-[9].

With the emergence of several potential applications, sources that emit terahertz radiation have also recently become a "hot" research topic. These include medical imaging [10] (including dental and skin cancer), biological weapons detection [11], explosives detection [12], gas sensing, pollution monitoring, and molecular spectroscopy [13].

The paper is organized as follows. Section II deals with some of the issues particularly pertinent to growth in a strained material system such as $\mathrm{Si} / \mathrm{SiGe}$. This is one of the main constraining factors in the design of $\mathrm{Si} / \mathrm{SiGe}$ active regions. Section III covers resonant tunneling in p-type $\mathrm{Si} / \mathrm{SiGe}$. Resonant tunneling through the barriers separating subsequent quantum wells in a quantum cascade active region controls the transport of carriers across the heterostructure. The amount of carriers that can travel through the active region has a direct effect on the gain. Section IV covers electroluminescence measurements at terahertz frequencies from $\mathrm{Si} / \mathrm{SiGe}$ cascade structures. The amount of gain in any laser depends on the lifetimes of the upper and lower laser levels. In Section V, lifetime measurements performed on $\mathrm{Si} / \mathrm{SiGe}$ quantum wells are discussed. Another important aspect regarding gain is how much of the optical mode overlaps with the active region. Conventional semiconductor lasers have exploited the refractive index step between epitaxial layers with different atomic mole fractions to achieve a high mode overlap in the vertical (growth) direction. In contrast, at terahertz frequencies, it is necessary to exploit surface-plasmon-enhanced guiding in order to achieve the same effect. Section VI explores the possibility of using buried silicide layers to obtain a high mode overlap in the vertical direction. Electroluminescence measurements in $\mathrm{Si} / \mathrm{SiGe}$ quantum cascade structures so far have exhibited wide $(>10 \mathrm{meV})$ intersubband emission peaks. Narrow peaks in the emission spectrum of some samples have been attributed to dopant impurities such as boron and phosphorus. Section VII discusses whether these impurities transitions might themselves 

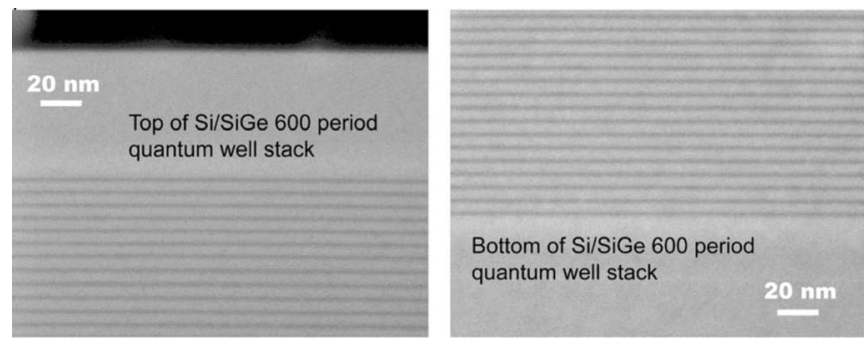

Fig. 1. TEM showing both the top and bottom few epilayers of a 600-period, $4-\mu \mathrm{m}$-thick, quantum well stack grown by gas source MBE.

be exploited to realize an electroluminescent terahertz siliconbased laser.

\section{MAterial Growth IsSUeS}

While a III-V terahertz quantum cascade laser has already been demonstrated [1], a silicon-based quantum cascade laser is yet to be realized. This has a lot to do with inherent difficulties of the less mature strained $\mathrm{Si} / \mathrm{SiGe}$ growth system, the chemistry of dopant materials, and the band structure of the system [14]. All $\mathrm{Si} / \mathrm{SiGe}$ quantum cascade emitter designs produced to date have been p-type using holes as the unipolar carriers. This is due to the large effective mass for electrons tunneling in the growth direction of Si $\left(\begin{array}{lll}1 & 0 & 0\end{array}\right)$ being $0.94 m_{\mathrm{e}}$, where $m_{\mathrm{e}}$ is the free electron mass [14]. This would result in the requirement of subnanometer tunnel barriers to allow significant tunneling through potential barriers. Also, most n-type dopants surface segregate, making the doping of the thin n-type layers difficult without doping layers grown after the As or $\mathrm{P}$ has been released in the growth chamber.

Typically the critical thickness at which dislocations occur is several orders of magnitude smaller than the necessary thickness of a quantum cascade active region and strain symmetrization is required [14]. The thickness and mole fractions of each successive $\mathrm{SiGe}$ epilayer are carefully adjusted so that each epilayer under compressive strain has neighboring epilayers under tensile strain, so that the average strain across the entire structure is zero. Strain does not have to be balanced over each individual pair of compressive and tensile layers but must be balanced over a distance less than the critical thickness. Therefore, typically the strain is balanced over a cascade period. To date we have demonstrated the growth of over $4 \mu \mathrm{m}$ of quantum cascade active region that has been perfectly strain balanced. A good example of this is shown in Fig. 1. This figure shows a transmission electron micrograph (TEM) of few of both the top and bottom epilayers of a 600 -period, 4- $\mu \mathrm{m}$-thick, quantum well stack grown by gas source molecular beam epitaxy (MBE) on a relaxed $\mathrm{Si}_{0.8} \mathrm{Ge}_{0.2}$ virtual substrate. By comparing the thicknesses of the epilayers in both the left- and the right-hand TEM pictures it is easy to see that the layers are uniform after $4 \mu \mathrm{m}$ of growth. This is a necessary condition for the active region of a real quantum cascade laser.

\section{RESONANT TunNELING IN P-TyPe SiGe}

The principle of the quantum cascade relies heavily on the basic quantum mechanical principle of tunneling, i.e., a particle

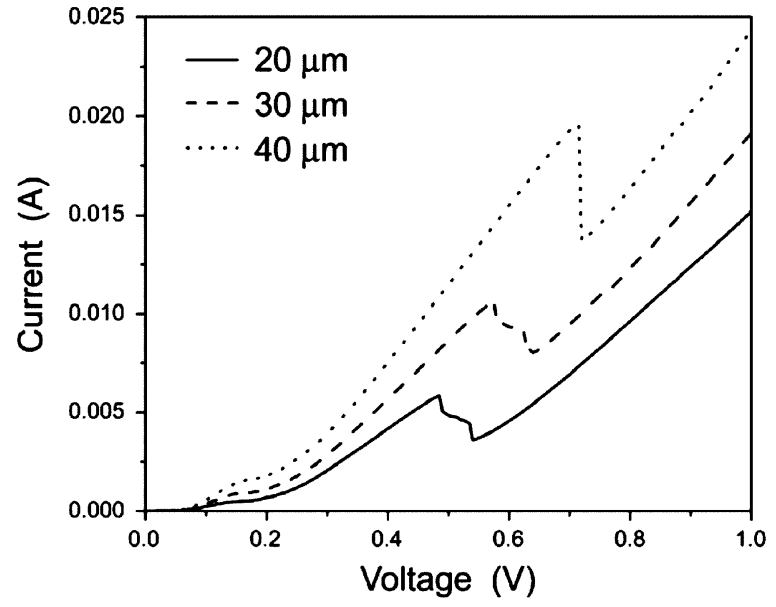

Fig. 2. Current versus voltage for three different RTD devices with different areas. Current peak corresponds to the tunneling between the contacts and the $\mathrm{HH} 2$ states in the quantum well.

may tunnel through a finite potential energy barrier even though it may not classically have enough energy to otherwise surmount the barrier. Resonant tunneling occurs when two energy states on either side of a potential energy barrier have the same energy. Resonant tunneling in a quantum cascade laser controls the transport of carriers across the heterostructure, and therefore has a direct effect on the gain. Significant understanding of the tunneling processes can be obtained from two tunnel barriers with a single quantum well sandwiched in between. Such a device is called a resonant tunneling diode (RTD).

There are many reports of $\mathrm{Si} / \mathrm{SiGe}$ RTDs in the literature especially p-type grown pseudomorphically on bulk silicon substrates [15], but relatively few reports on high-quality strain-relaxed buffers [16], [17]. In particular, there are very few examples of strain symmetrized p-type RTDs with energy level separations at terahertz frequencies. A number of wafers were grown to gain an understanding of tunneling of holes in $\mathrm{Si} / \mathrm{SiGe}$ structures with the same composition and barriers as designs for terahertz cascades, and the results from one wafer are presented here. The epitaxial layer structure consisted of a 4-nm i-Si $\mathrm{Si}_{0.6} \mathrm{Ge}_{0.4}$ quantum well sandwiched between 4-nm i-Si barriers. Either side of the Si barriers had $11 \mathrm{~nm}$ of graded i-Si $\mathrm{Si}_{x} \mathrm{Ge}_{1-x}$ leading to an $\mathrm{Si}_{0.8} \mathrm{Ge}_{0.2}$ ohmic contact layer doped at a concentration greater than $10^{19} \mathrm{~cm}^{-3}$. The entire structure just described was grown on top of a $\mathrm{Si}_{0.8} \mathrm{Ge}_{0.2}$ virtual substrate. Mesas were etched using $\mathrm{SiCl}_{4}$ reactive ion etching and $\mathrm{Al}(1 \% \mathrm{Si})$ evaporated to form shallow ohmic contacts. A rapid thermal anneal below $400^{\circ} \mathrm{C}$ was performed to prevent spiking of the contacts. A thick $\mathrm{SiO}_{x}$ layer was used to isolate the top and bottom contacts before a via hole was used to contact the top ohmic contact of the device. Fig. 2 shows typical current-voltage curves for the three mesa sizes processed from a single wafer. In each case, a large nonlinearity is observed at around $0.5 \mathrm{~V}$ indicating resonant tunneling between the ohmic contacts and the heavy-hole $2(\mathrm{HH} 2)$ state in the quantum well. 


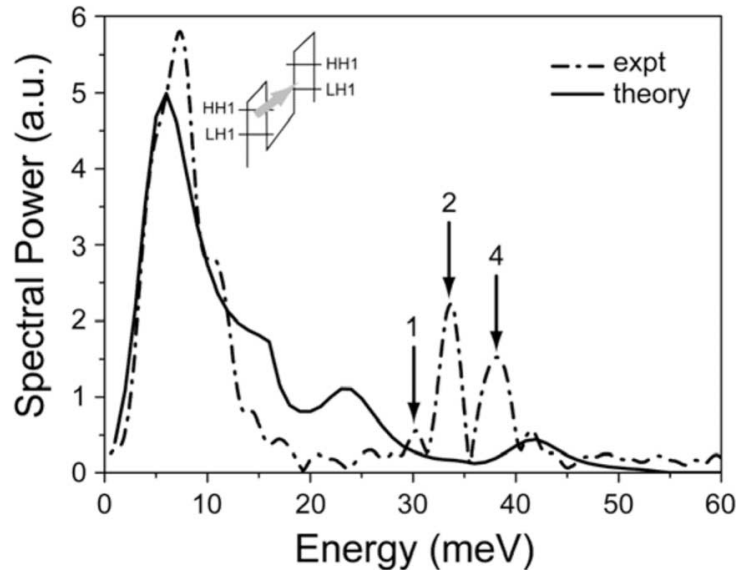

Fig. 3. TE-polarized edge emission spectrum taken under an applied bias of $6.4 \mathrm{~V}(10 \%$ duty cycle) at $4.2 \mathrm{~K}$ (dashed line) and theoretical predictions (solid line). The features marked 1,2, and 4 are the well-known $\mathrm{p}_{3 / 2}$ series of Si:B.

\section{TERAHERTZ ELECTROLUMINESCENCE FROM SiGe CASCADES}

The first demonstration of intersubband electroluminescence from a $\mathrm{Si} / \mathrm{SiGe}$ cascade structure was at midinfrared frequencies. It exploited an $\mathrm{HH}$-to-HH intersubband transition [2] using pseudomorphically grown SiGe quantum wells on a bulk silicon substrate. The disadvantage of the pseudomorphic growth is that only a small number of quantum wells may be grown, up to a metastable critical thickness [14], which is too small to achieve sufficient active cascade periods for large enough gain for a laser. The first demonstration of terahertz electroluminescence from a Si/SiGe strain symmetrized structure was reported in [3]. This electroluminescent emission, however, resulted from applying electric fields along the quantum well of modulationdoped p-type $\mathrm{Si} / \mathrm{SiGe}$ quantum wells, thereby heating holes to higher states, and emitting photons when the excited holes were relaxed to the ground state. The first demonstration of terahertz electroluminescence from a real $\mathrm{Si} / \mathrm{SiGe}$ quantum cascade structure was demonstrated by a subset of the authors of this paper [3]. This structure contained 30 active periods consisting of a quantum well and a barrier arranged in a so-called quantum staircase structure. It is difficult, however, to attain the population inversion required to produce a laser in this type of structure. This is because the tunneling rate controlling carrier injection into the upper energy level will be nominally the same as the tunneling rate controlling the depopulation of the lower energy into the next adjacent well. More recently, however, the same authors demonstrated terahertz electroluminescence from a 100-period interwell, or diagonal structure [4]. It is easier to obtain population inversion in this type of structure, since the tunneling rates can now be tuned by altering the electric field across the structure. This type of structure also has the added advantage that the transition energies can be tuned with the applied electric field.

Figs. 3 and 4 show the experimentally measured (dashed line) and theoretically calculated (solid line) TE- and TM-polarized edge emission spectrum at $4.2 \mathrm{~K}$, respectively. The theoretical calculations were performed using a six-band $\mathbf{k} \cdot \mathbf{p}$ model with self-consistent inclusion of the internal charge density. Exact

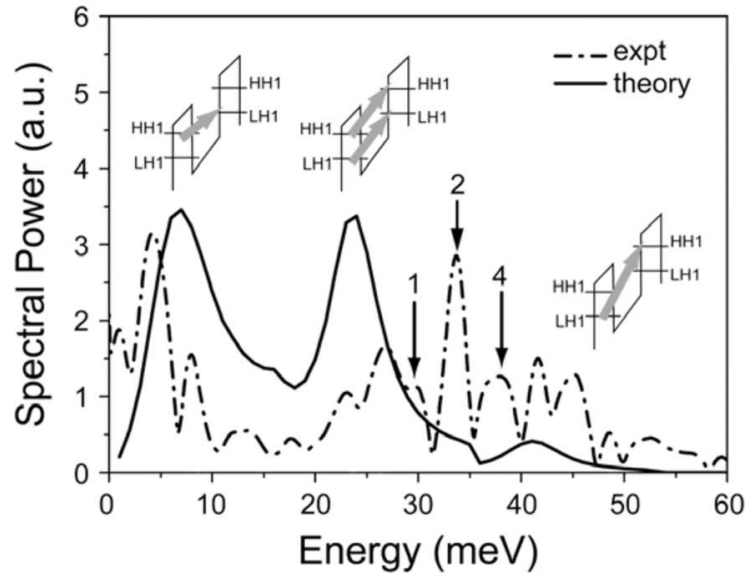

Fig. 4. TM-polarized edge emission spectrum taken under an applied bias of $6.4 \mathrm{~V}(10 \%$ duty cycle) at $4.2 \mathrm{~K}$ (dashed line) and theoretical predictions (solid line). The features marked 1, 2, and 4 are the well-known $\mathrm{p}_{3 / 2}$ series of Si:B.

details of these calculations are published elsewhere [18]. Fig. 3 (TE) shows one well-defined spectral feature centered on $8 \mathrm{meV}$, attributed to the lowest energy HH to light-hole (LH) interwell transition, HH1-LH1. It is very difficult to completely rule out thermal origins for this feature (black body). However, since HH-LH or LH-HH transitions allow TE-polarized intersubband radiative transitions, while for $\mathrm{HH}-\mathrm{HH}$, LH-LH, or electron-toelectron radiative intersubband transitions only TM polarization can be observed at small $k$-parallel, it should be possible to observe a discernible difference between the two spectra. This is indeed the case. In the TM spectrum shown in Fig. 4, the 8-meV feature is strongly suppressed while an additional feature between 20 and $30 \mathrm{meV}$ is clearly observed. This feature is not present in the TE spectrum and is identified as the degenerate LH1-LH1 and HH1-HH1 interwell transition. Between 30 and $40 \mathrm{meV}$, three sharp features are observed labeled 1, 2, and 4. These correspond to the well known $\mathrm{p}_{3 / 2}$ series of Si:B [19], [20]. The considerable differences between Figs. 3 and 4 along with their good agreement with theoretical predictions provide strong evidence that these features do indeed result from intersubband transitions. Further evidence can be found in [4], where the frequency shift of these peaks with applied bias is discussed.

The best theoretical fits to the experimental emission spectra require population inversion to be present in the calculations. In contrast, there is poor agreement between experimental and theoretical spectra if thermal equilibrium populations are used. The theoretical modeling therefore suggests that population inversion is obtained in these samples although there is no experimental proof to confirm this prediction.

\section{INTERSUBBAND LIFETIME MEASUREMENTS}

In order to produce gain in a potential laser material, it is necessary to achieve population inversion. This typically happens when the upper and lower states in the radiative transition have different lifetimes, specifically when the upper laser level has a long lifetime, and the lower laser level has a short lifetime. Using FELIX, the free electron laser facility at Utrecht in the Netherlands, a number of pump-probe experiments were 


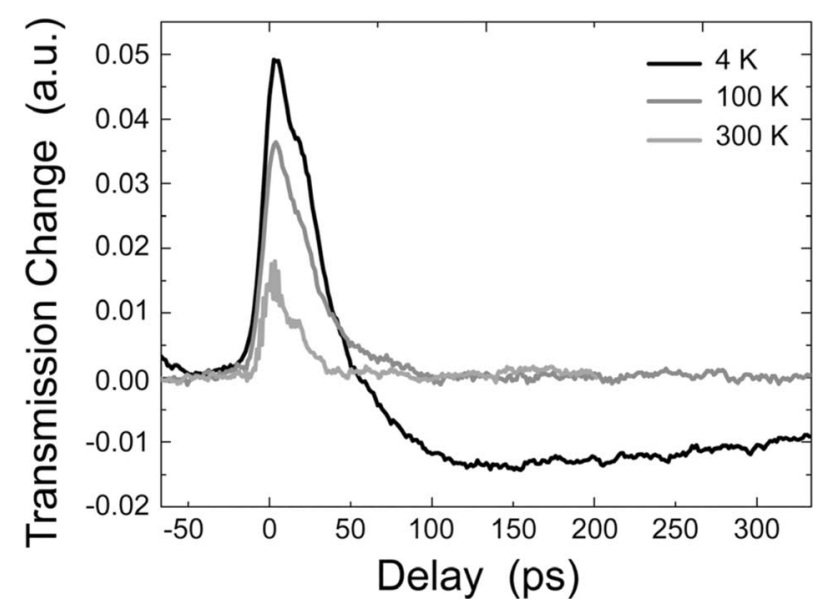

Fig. 5. Differential probe transmission against optical delay at three different temperatures for the LH1-to-HH1 intrawell lifetime at $14.7 \mathrm{meV}$ $(3.53 \mathrm{THz})$

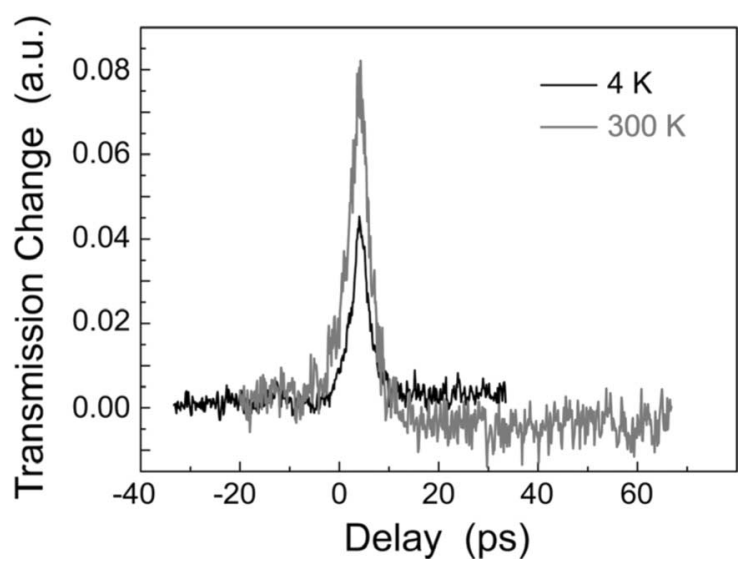

Fig. 6. Differential probe transmission against optical delay for two temperatures for the LH1-to-HH1 intrawell lifetime at $32.9 \mathrm{meV}(7.90 \mathrm{THz})$.

performed to determine the upper state lifetime of SiGe quantum wells that were engineered to have an intrawell LH1-to$\mathrm{HH} 1$ energy level spacing at terahertz frequencies. Full details of the band structure and experimental details can be found elsewhere [21], [22]. Fig. 5 shows the differential probe transmission against optical delay for three temperatures between 4.2 and 300 $\mathrm{K}$. From this measurement, it is observed that lifetimes as long as $25 \mathrm{ps}$ persist for temperatures up to $300 \mathrm{~K}$. More importantly, this measurement shows that the lifetime remains relatively constant over this large temperature range due to the lack of polar optical phonon scattering in Group IV materials [18]. The intersubband lifetimes decrease as the energy is increased toward the $\mathrm{Ge}-\mathrm{Ge}$ optical phonon energy as this mechanism becomes the dominant nonradiative process. This is in stark contrast to what occurs in the III-V material system. Here, the lifetime rapidly collapses to subpicosecond values above $40 \mathrm{~K}$ due to polar optical phonon scattering. This provides strong evidence that any successful $\mathrm{Si} / \mathrm{SiGe}$ quantum cascade laser operating below the optical phonon energy has the potential to operate over a wider temperature range than its III-V counterparts.

Once the pump energy is increased to larger values, the intersubband lifetime rapidly collapses below the resolution limit of

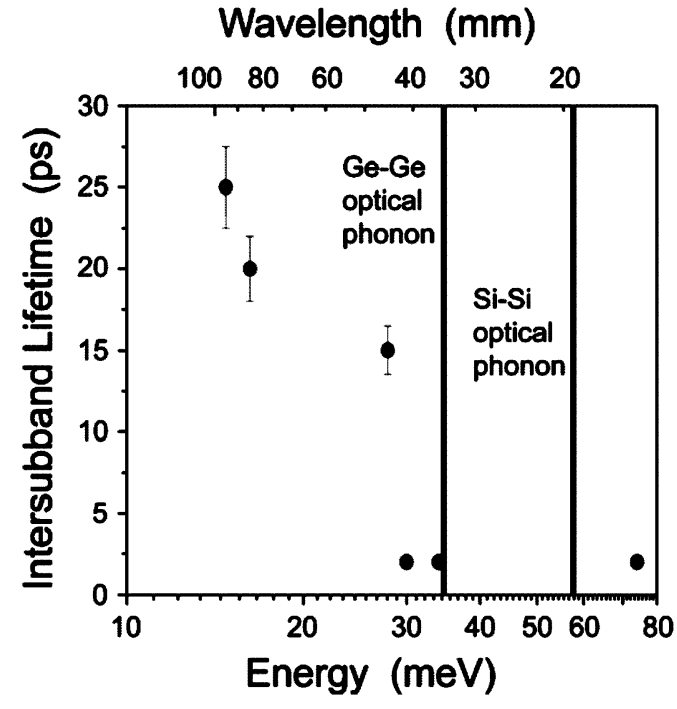

Fig. 7. Plot of intersubband lifetime for an intrawell LH1-to-HH1 transition against both energy (bottom axis) and wavelength (top axis). The three points above $30 \mathrm{meV}$ are resolution limited in the experiment so that the values are below the probe width of 2 ps.

the measurement technique of 2 ps. Fig. 6 shows the differential probe transmission at $32.9 \mathrm{meV}$, comparable to the $\mathrm{Ge}-\mathrm{Ge}$ optical phonon energy of $37 \mathrm{meV}$. This is entirely expected, since as the Ge-Ge phonon energy is approached, carriers relax to the ground state by the nonradiative optical phonon scattering mechanism. The situation is summarized in Fig. 7, which shows the intrawell LH1-to-HH1 intersubband lifetime plotted against energy. The lifetimes can be observed to decrease with increasing energy, and at $30 \mathrm{meV}$ are limited by the measurement resolution of the pump-probe technique. The Ge-Ge and $\mathrm{Si}-\mathrm{Si}$ phonon energies are highlighted for clarification.

\section{BURIED SILICIDE WAVEGUIDES}

Realizing an active region that exhibits large material gain is of course like winning only half the battle. If the electric field of the propagating electromagnetic mode cannot interact with the material, then no laser can be produced. The magnitude of this interaction is described by the modal overlap. In conventional visible and near infrared semiconductor lasers, this is achieved by engineering a large refractive index step around the active region in order to tightly confine the electromagnetic mode. Unfortunately, as the wavelength gets longer it becomes progressively harder to engineer a significant refractive index step. One of the key enabling technologies in the development of the III-V terahertz quantum cascade laser was the introduction of surface plasmon waveguides [1]. Surface plasmon waves form at the interface between metallic and dielectric media. In the case of the III-V cascade laser, sandwiching the active region between the top Au-doped semiconductor ohmic contact and a bottom highly doped semiconductor layer forms a double plasmon waveguide. For the bottom doped-semiconductor layer to become a reflector, the doping of the layer must be made sufficiently high to result in a negative dielectric constant. As higher doping will result in a better reflector but also will increase the 


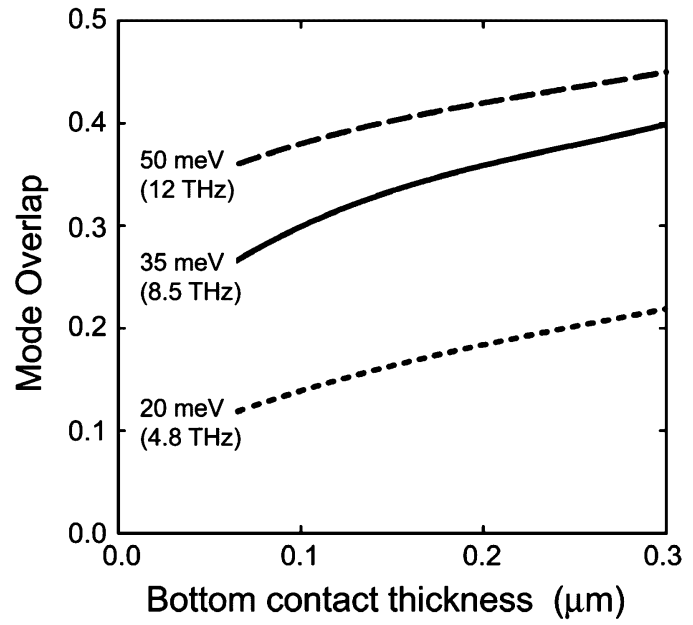

Fig. 8. Plot showing the mode overlap against bottom contact thickness for a $4-\mu \mathrm{m}$ active region at three different frequencies.

waveguide losses due to free carrier absorption, the doping density is selected to allow a compromise between modal overlap and waveguide loss.

In silicon, however, the mobility is much lower than in the corresponding III-V material system. This means that the conductivity is also much lower. Unfortunately, a high conductivity is required for the imaginary part of the dielectric constant to become larger than the real part. This is necessary for a negative dielectric constant and a good reflector. For this reason, a heavily doped $\mathrm{Si}$ or $\mathrm{SiGe}$ layer will not act as a good plasmon reflector unlike $\mathrm{n}^{+} \mathrm{GaAs}$ in the III-V laser. Assuming a $4-\mu$ m-thick SiGe active region sandwiched between a top Al-p ${ }^{++}-\mathrm{SiGe}$ ohmic contact and a bottom contact layer with a realistic doping density of $3 \times 10^{20} \mathrm{~cm}^{-3}$, it is possible to calculate the mode overlap as a function of bottom contact layer thickness (Fig. 8) [23]. This is a reasonable approximation of the quantum cascade structures that have been grown, fabricated, and characterized to date with active regions of up to $4-\mu \mathrm{m}$ thickness on top of $\sim 3.5-\mu \mathrm{m}$ virtual substrates. Fig. 8 shows that this configuration provides a waveguide with very poor modal overlap, with a best case scenario of between $10 \%$ and $20 \%$ overlap at $4.8 \mathrm{THz}$.

The situation can be dramatically improved if advantage is taken of a technology that is only available in the $\mathrm{Si}$ (and $\mathrm{SiGe}$ ) material system, i.e., replacing the bottom doped-contact layer with a buried silicide layer. The silicide layer has the advantage of having a much higher electrical conductivity than a semiconductor, and typically within one order of magnitude of good metals. A full description of the fabrication of a buried silicide layer can be found elsewhere [24]. The four most mature silicide technologies are $\mathrm{WSi}_{2}, \mathrm{CoSi}_{2}, \mathrm{TiSi}_{2}$, and NiSi. So far, we have concentrated on $\mathrm{WSi}_{2}$ because the low-resistivity phase is stable to temperatures over $800^{\circ} \mathrm{C}$ allowing $\mathrm{Si}$ and $\mathrm{SiGe}$ heterolayer growth after a silicide has been formed. Fig. 9 shows the mode overlap for a $4-\mu \mathrm{m}$-thick SiGe active region, sandwiched between a top $\mathrm{Al}-\mathrm{p}^{++}-\mathrm{SiGe}$ ohmic contact and a bottom $\mathrm{WSi}_{2}$ contact layer, as a function of silicide thickness [23]. The dra-

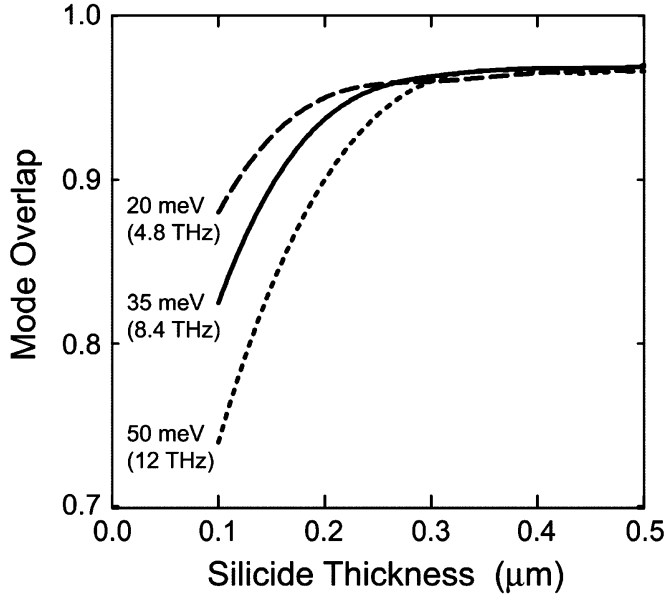

Fig. 9. Plot showing the mode overlap against buried silicide $\left(\mathrm{WSi}_{2}\right)$ thickness for a $4-\mu \mathrm{m}$ active region at three different frequencies.

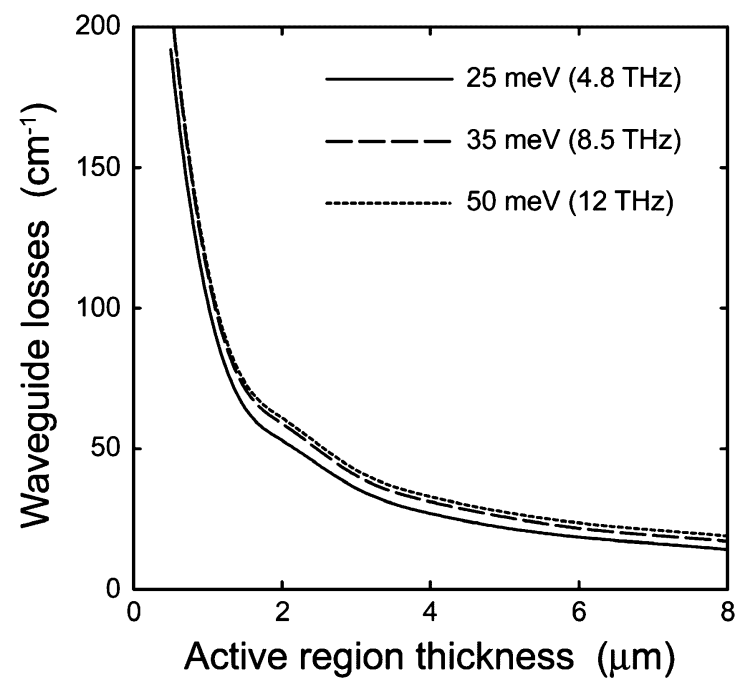

Fig. 10. Plot showing the waveguide losses against active region thickness on top of a $400-\mathrm{nm} \mathrm{WSi}_{2}$ reflector for three different frequencies.

matic improvement in the situation is quite noticeable. Using a buried silicide of thickness $0.3 \mu \mathrm{m}$ or larger, the overlap is now approximately $95 \%$ at $4.8 \mathrm{THz}$.

Fig. 10 shows the calculated waveguide losses as a function of active region thickness for $\mathrm{WSi}_{2}$. Again it is assumed that the active region is sandwiched between a top $\mathrm{Al}-\mathrm{p}^{++}-\mathrm{SiGe}$ ohmic contact and a bottom $\mathrm{WSi}_{2}$ contact layer. Once the active region thickness becomes larger than $4 \mu \mathrm{m}$, the losses become quite small and certainly comparable to the waveguide losses and modal overlaps quoted in terahertz GaAs quantum cascade lasers [1].

Fig. 11 shows a TEM of a wafer we have grown with a buried $\mathrm{WSi}_{2}$ layer wafer [24] to produce high modal overlap. It consists of $4.005 \mu \mathrm{m}$ of $\mathrm{SiGe}$ quantum cascade active region, preceded by a $0.990-\mu$ m-thick $\mathrm{Si}_{0.8} \mathrm{Ge}_{0.2}$ constant composition layer, a 2.830- $\mu \mathrm{m}$ graded $\mathrm{Si}_{x} \mathrm{Ge}_{1-x}$ buffer, and a $1.280-\mu \mathrm{m}$-thick Si layer, on top of a $440-\mathrm{nm}$-thick $\mathrm{WSi}_{2}$ layer. 


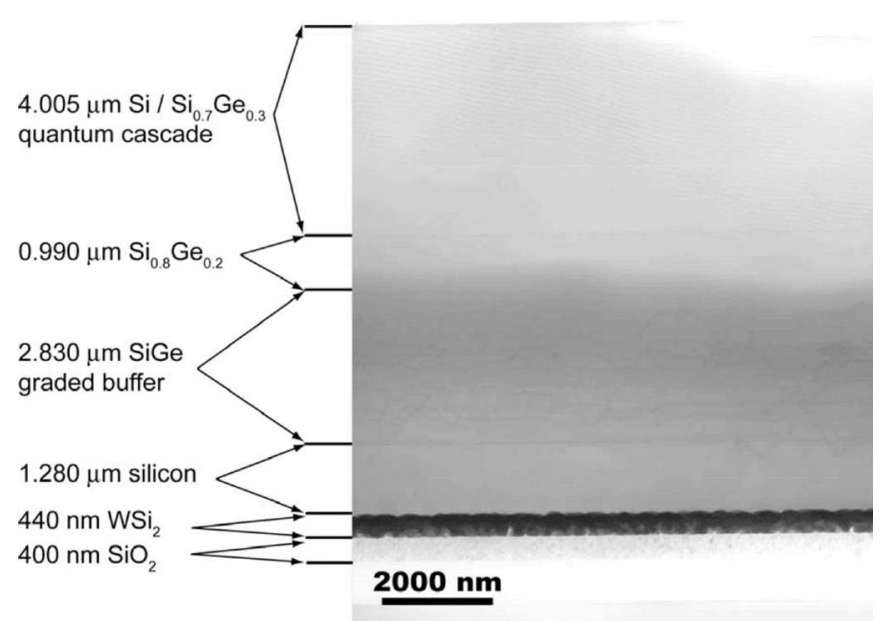

Fig. 11. TEM showing a 600-period, $4-\mu \mathrm{m}$-thick quantum well stack grown by gas source MBE on top of a $400-\mathrm{nm}$ buried $\mathrm{WSi}_{2}$ layer.

\section{IMPURITY-BASED TERAHERTZ ELECTROLUMINESCENCE}

In Section IV, it was noted that between 30 and $40 \mathrm{meV}$ three sharp features are observed in both the TE-polarized (Fig. 3) and the TM-polarized (Fig. 4) electroluminescence spectra. These transitions were attributed to the well known $\mathrm{p}_{3 / 2}$ series of Si:B [19], [20]. The conventional wisdom has been that these transitions are excited by impact ionization, though recent work (to be published) has shown that thermal effects may play a more important role. Under certain bias conditions and at the lowest temperatures in some structures, the intersubband transition can be suppressed and the terahertz electroluminescence arises purely from the impurity transitions [5], [9].

The possibly of harnessing these transitions to make an impurity-based laser has generated considerable excitement over the past few years. The first of these impurity-based lasers was demonstrated in 2002 [25]. A pulsed $\mathrm{CO}_{2}$ pump laser was used to excite electrons high into the conduction band. These electrons rapidly relax into the upper $2 \mathrm{p}_{0}$ lasing state via intervalley acoustic and optical phonon scattering. The long lifetime of the $2 \mathrm{p}_{0}$ state causes a relaxation bottleneck, and leads to a population inversion. Stimulated emission on the $2 \mathrm{p}_{0} \rightarrow 1 \mathrm{~s}\left(T_{2}\right)$ transition at $22.4 \mathrm{meV}(5.41 \mathrm{THz})$ then occurs [26]. A similar mechanism was exploited in bismuth-doped silicon [27] (Si:Bi). Here, lasing transitions occur between the upper $2 \mathrm{p}_{ \pm}$ level and the closely spaced $1 \mathrm{~s}(E)$ and $1 \mathrm{~s}\left(T_{2}\right)$ lower levels. This results in two emission lines at $23.76 \mathrm{meV}(5.74 \mathrm{THz})$ and $25.49 \mathrm{meV}(6.16 \mathrm{THz})$. Lasing emission was also observed from similar $2 \mathrm{p}_{ \pm} \rightarrow 1 \mathrm{~s}(E)$ and $2 \mathrm{p}_{ \pm} \rightarrow 1 \mathrm{~s}\left(T_{2}\right)$ transitions in arsenic-doped silicon (Si:As) [28]. This time the emission lines were at $24.94 \mathrm{meV}(6.03 \mathrm{THz})$ and $26.33 \mathrm{meV}(6.36 \mathrm{THz})$. Antimony-doped silicon ( $\mathrm{Si}: \mathrm{Sb})$ was also shown to exhibit lasing [29]. One laser emission line at $24.07 \mathrm{meV}$ (5.15 THz from the $2 \mathrm{p}_{0} \rightarrow 1 \mathrm{~s}\left(T_{2}\right)$ transition is observed.

Therefore, an obvious question is: Can such impurity transitions be used to produce an electrically pumped laser? Fig. 12 shows two terahertz electroluminescence spectra for a sample containing boron and another containing phosphorus. All elec-

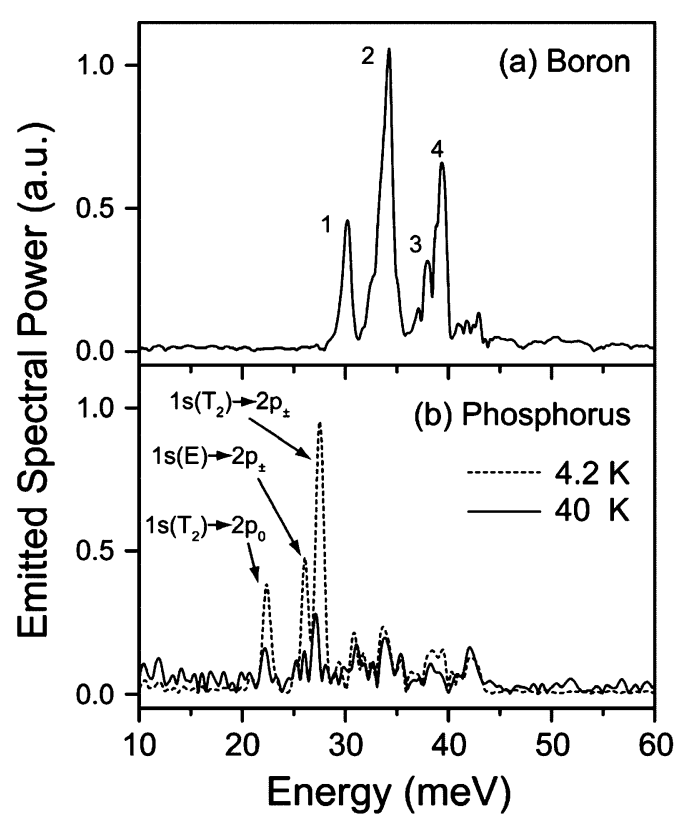

Fig. 12. Terahertz electroluminescence spectra for two samples. (a) Sample containing boron. (b) Sample containing phosphorus.

troluminescence measurements so far suggested that the appearance of these transitions in the electroluminescence spectra was highly dependent on the temperature of holes in the device. This hole temperature in turn was dependent on both the heat-sink temperature (i.e., the temperature of the cold finger of the cryostat) and on the amount of current flowing through the particular device. In the case of a biased device, if sufficient current is flowing, then it can contribute to an additional rise in temperature through joule heating.

In order to properly assess the effect of temperature on the impurity transitions, it is necessary to consider a passive device with no current flowing. In the simplest case, this can be achieved by considering the effect of temperature on the absorption properties of a moderately doped bulk silicon wafer $\left(10^{15}-10^{16}\right.$ $\mathrm{cm}^{-3}$ ). Fig. 13 shows the absorption from a boron-doped silicon wafer with temperature at terahertz frequencies $(2.4-14.4 \mathrm{THz})$. At very low heat sink temperatures, three very sharp absorption features can be observed between 30 and $40 \mathrm{meV}$. These correspond to the lines 1,2 , and 4 of the well known $\mathrm{p}_{3 / 2}$ series of $\mathrm{Si}(\mathrm{B})$ [19], [20]. As the heat sink temperature is raised between 4.2 and $90 \mathrm{~K}$, the transitions become progressively weaker, until they are finally extinguished around $100 \mathrm{~K}$ [9]. This corresponds closely with a reduction in the total absorption up to $100 \mathrm{~K}$. In contrast, the behavior of the phosphorus-doped sample in Fig. 14 is quite different. Unlike the boron-doped sample, the maximum absorption of the phosphorus-doped sample is not at $4.2 \mathrm{~K}$, but at $50 \mathrm{~K}$. Close examination of Fig. 14 reveals that the absorption spectrum is dominated by three features in the range 30-45 $\mathrm{meV}$ at $4.2 \mathrm{~K}$. These correspond to the $2 \mathrm{p}_{0}, 2 \mathrm{p}_{ \pm}$, and $3 \mathrm{p}_{ \pm} \mathrm{Ly}-$ man series transitions to the ground state $1 \mathrm{~s}\left(A_{1}\right)$ [20]. As the heat sink temperature is increased, a further set of thermally activated absorption lines appear between 20 and $30 \mathrm{meV}$. By considering the binding energies in [19], it is possible to show that these transitions are between the $2 \mathrm{p}_{0}, 2 \mathrm{p}_{ \pm}$energy levels 


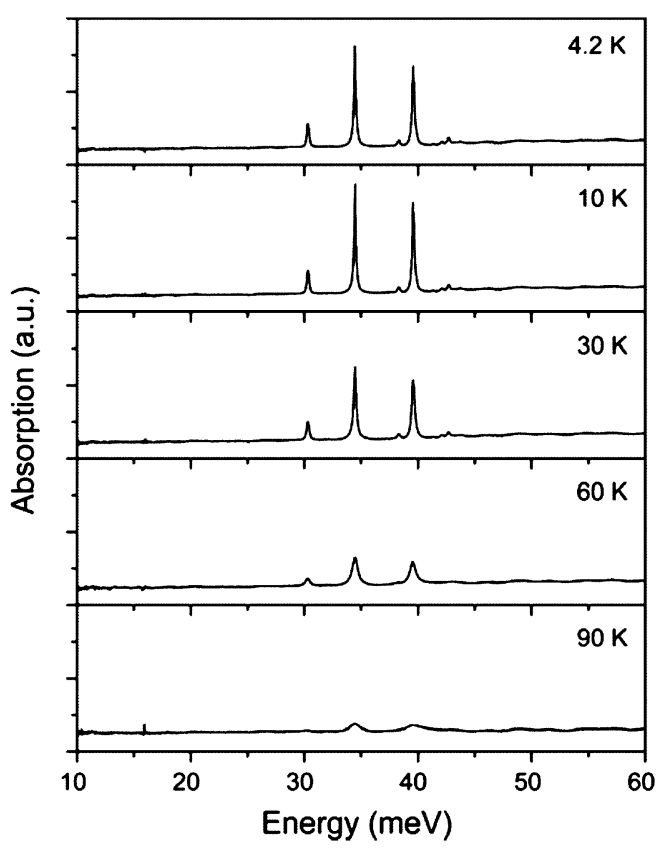

Fig. 13. Terahertz absorption from a boron-doped silicon wafer with temperature. The wafer has a resistivity of $10-20 \Omega \cdot \mathrm{cm}$, giving a boron concentration of $\sim 1 \times 10^{15} \mathrm{~cm}^{-3}$.

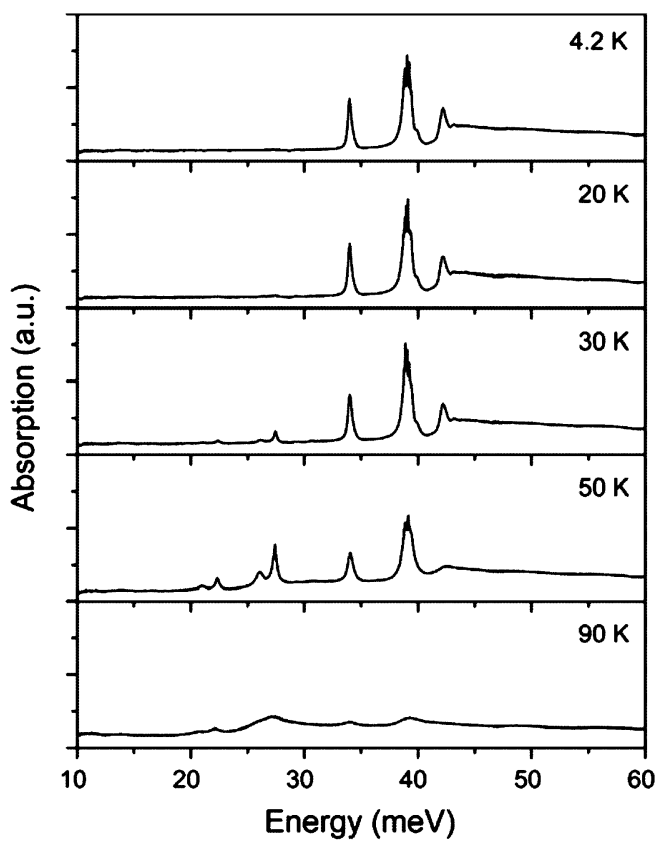

Fig. 14. Terahertz absorption from a phosphorus-doped silicon wafer with temperature. The wafer has a resistivity of $0.3 \Omega \cdot \mathrm{cm}$, giving a phosphorus concentration of $1.5 \times 10^{16} \mathrm{~cm}^{-3}$

and the excited ground state $1 \mathrm{~s}\left(T_{1}\right)$. These features reach maximum transition strength at $50 \mathrm{~K}$. It is from these additional thermally activated transitions that emission was observed in Fig. 12. Above $50 \mathrm{~K}$, the strength of all the transitions begins to weaken until they are extinguished at around $100 \mathrm{~K}$.

To summarize, the measured temperature dependence of these transitions shows that the most optimistic operating range of an impurity-based laser is $4.2 \mathrm{~K} \leq \mathrm{T} \leq 90 \mathrm{~K}$. This represents an ideal passive device with no current flowing. In a real device with current flowing and associated joule heating, the actual operating range is likely to be much narrower and it is unlikely such a device would have a maximum operating temperature above $40 \mathrm{~K}$ as in Fig. 12. This is quite consistent with the value quoted in [27]. The major problem with such impurity devices is the tradeoff between doping, current, gain, and free carrier losses. Initial estimates of the gain for electrically pumped impurity structures suggest that it is going to be difficult to overcome the waveguide losses with simple device structures.

\section{CONCLUSION}

In this paper, we have discussed several aspects pertinent to the realization of an electrically pumped silicon-based terahertz laser. Growth in a strained material system such as $\mathrm{Si} / \mathrm{SiGe}$ has been discussed. It has been shown that it is possible to grow a low dislocation density, thick multiperiod $\mathrm{Si} / \mathrm{SiGe}$ superlattice, with good layer thickness uniformity. Quantum mechanical tunneling between valance band states in p-type Si/SiGe RTD structures has been demonstrated, which is a prerequisite for the operation of a p-type $\mathrm{Si} / \mathrm{SiGe}$ quantum cascade laser. Lifetime measurements in quantum wells with an energy spacing at terahertz frequencies have also been performed. These measurements show that the lifetime remains relatively constant over a large temperature range and provides strong evidence that a successful SiGe quantum cascade laser has the potential for operation over a wider temperature range than its III-V counterpart. The question of high vertical confinement in silicon has been addressed and it has been shown that buried silicide technology provides a possible route forward. Modeling suggests that modal overlap and waveguide losses comparable to GaAs terahertz quantum cascade lasers can be produced. Finally, the possibility of exploiting impurities such as boron and phosphorus to realize an electrically pumped terahertz siliconbased laser has been explored. Absorption measurements show that it is unlikely such an impurity-based device would have a maximum operating temperature above $40 \mathrm{~K}$. The impressive results from optically pumped $\mathrm{Si}$-impurity lasers demonstrate that lasers can be produced with these impurities although the additional free carrier losses from higher doping levels required to reduce resistive joule heating and increase gain make the realization of an electrically pumped silicon-impurity laser far more difficult than the optically pumped version.

In summary, all the necessary individual components to realize a terahertz quantum cascade laser are in place. The challenge, which remains, is to design and grow a quantum cascade active region that exhibits sufficient gain to provide lasing action.

\section{REFERENCES}

[1] R. Kohler, A. Tredicucci, F. Beltram, H. E. Beere, E. H. Linfield, A. G. Davies, D. A. Ritchie, R. C. Iotti, and F. Rossi, "Terahertz semiconductor-heterostructure laser," Nature, vol. 417, no. 6885, pp. 156159, May 2002.

[2] G. Dehlinger, L. Diehl, U. Gennser, H. Sigg, J. Faist, K. Ensslin, D. Grützmacher, and E. Müller, "Intersubband electroluminescence from silicon-based quantum cascade structures," Science, vol. 290, pp. 22772280, Dec. 2000 
[3] S. A. Lynch, R. Bates, D. J. Paul, D. J. Norris, A. G. Cullis, Z. Ikonic, R. W. Kelsall, P. Harrison, D. D. Arnone, and C. R. Pidgeon, "Intersubband electroluminescence from $\mathrm{Si} / \mathrm{SiGe}$ cascade emitters at terahertz frequencies," Appl. Phys. Lett., vol. 81, no. 9, pp. 1543-1545, Aug. 2002.

[4] R. Bates, S. A. Lynch, D. J. Paul, Z. Ikonic, R. W. Kelsall, P. Harrison, S. L. Liew, D. J. Norris, A. G. Cullis, W. R. Tribe, and D.D. Arnone, "Interwell intersubband electroluminescence from $\mathrm{Si} / \mathrm{SiGe}$ quantum cascade emitters," Appl. Phys. Lett., vol. 83, no. 20, pp. 4092-4094, Nov. 2003.

[5] S. A. Lynch, S. S. Dhillon, R. Bates, D. J. Paul, D. D. Arnone, D. J. Robbins, Z. Ikonic, R. W. Kelsall, P. Harrison, D. J. Norris, A. G. Cullis, C. R. Pidgeon, P. Murzyn, and A. Loudon, "Si-based electroluminescence at THz frequencies," J. Mater. Sci. B, vol. 89, p. 12, 2002.

[6] T. N. Adam, R. T. Troeger, S. K. Ray, P.-C. Lv, and J. Kolodzey, "Terahertz electroluminescence from boron-doped silicon devices," Appl. Phys. Lett., vol. 83, no. 9, pp. 1713-1715, Sep. 2003.

[7] P.-C. Lv, R. T. Troeger, T. N. Adam, S. Kim, J. Kolodzey, I. N. Yassievich, M. A. Odnoblyudov, and M. S. Kagan, "Electroluminescence at $7 \mathrm{THz}$ from phosphorus donors in silicon," Appl. Phys. Lett., vol. 85, no. 1, p. 22, Jul. 2004

[8] P.-C. Lv, R. T. Troeger, S. Kim, S. K. Ray, K. W. Goossen, J. Kolodzey, I. N. Yassievich, M. A. Odnoblyudov, and M. S. Kagan, "Terahertz emission from electrically pumped gallium doped silicon devices," Appl.Phys. Lett., vol. 85, no. 17, pp. 3660-3662, Oct. 2004.

[9] S. A. Lynch, P. Townsend, G. Matmon, D. J. Paul, M. Bain, H. S. Gamble, J. Zhang, Z. Ikonic, R. W. Kelsall, and P. Harrison, "Temperature dependence of terahertz optical transitions from boron and phosphorus dopant impurities in silicon," Appl. Phys. Lett., vol. 87, pp. 101114-1101114-3, Sep. 2005.

[10] D. D. Arnone, C. M. Ciesla, and M. Pepper, "Terahertz imaging comes into view," Phys. World, vol. 13, no. 4, pp. 35-40, Apr. 2000.

[11] L. A. Vanderberg, "Detection of biological agents: Looking for bugs in all the wrong places," Appl. Spectrosc., vol. 54, p. 376A, 2000.

[12] Y. C. Shen, T. Lo, P. F. Taday, B. E. Cole, W. R. Tribe, and M. C. Kemp, "Detection and identification of explosives using terahertz pulsed spectroscopic imaging," Appl. Phys. Lett., vol. 86, no. 241116, 2005.

[13] F. N. Keutsch, M. G. Brown, P. B. Petersen, R. J. Saykally, M. Geleijns, and A. Van der Avoird, "Terahertz vibration-rotation-tunneling spectroscopy of water clusters in the translational band region of liquid water," J. Chem. Phys., vol. 114, pp. 3994-4004, 2001.

[14] D. J. Paul, "Si/SiGe heterostructures: From material and physics to devices and circuits," Semicond. Sci. Technol., vol. 19, no. 10, pp. R75-R108, Oct. 2004.

[15] D. X. Xu, G. D. Shen, M. Willander, G. V. Hansson, J. F. Luy, and F. Schäffler, "Variations of resonant tunneling properties with temperature in strained $\mathrm{Si}_{1-x} \mathrm{Ge}_{x} \mathrm{Si}$ double barrier structures," Appl. Phys. Lett., vol. 58, no. 22, pp. 2500-2502, Jun. 1991.

[16] P. See and D. J. Paul, "The scaled performance of $\mathrm{Si} / \mathrm{Si}_{1-x} \mathrm{Ge}_{x}$ resonant tunneling diodes," IEEE Electron Device Lett., vol. 22, no. 12, pp. 582584, Dec. 2001

[17] S. Tsujino, S. Mentese, L. Diehl, E. Müller, B. Haas, D. Bächle, S. Stutz, D. Grützmacher, Y. Campidelli, O. Kermarrec, and D. Bensahel, "Resonant tunneling in Si-Ge superlattices on relaxed buffer substrates," Appl. Surf. Sci., vol. 224, pp. 377-381, 2004.

[18] Z. Ikonic, P. Harrison, and R. W. Kelsall, "Intersubband hole-phonon and alloy disorder scattering in SiGe quantum wells," Phys. Rev. B, Condens. Matter, vol. 64, no. 245311, 2001.

[19] C. Jagannath, Z. W. Grabowski, and A. K. Ramdas, "Linewidths of the electronic spectra of donors in silicon," Phys. Rev. B, Condens. Matter, vol. 23, no. 5, pp. 2082-2098, Mar. 1981.

[20] A. K. Ramdas and S. Rodriguez, "Spectroscopy of the solid-state analogues of the hydrogen atom: Donors and acceptors in semiconductors," Rep. Prog. Phys., vol. 44, pp. 1297-1387, 1981.

[21] P. Murzyn, C. R. Pidgeon, J.-P. R. Wells, I. V. Bradley, Z. Ikonic, R. W. Kelsall, P. Harrison, S. A. Lynch, D. J. Paul, D. D. Arnone, D. J. Robbins, D. Norris, and A. G. Cullis, "Picosecond intersubband dynamics in p-Si/SiGe quantum well emitters," Appl. Phys. Lett., vol. 80, no. 8, pp. 1456-1458, Feb. 2002.

[22] R. W. Kelsall, Z. Ikonic, P. Murzyn, C. R. Pidgeon, P. J. Phillips, D. Carder, P. Harrison, S. A. Lynch, P. Townsend, D. J. Paul, S. L. Liew, D. J. Norris, and A. G. Cullis, "Intersubband lifetimes in p-Si/SiGe terahertz quantum cascade heterostructures," Phys. Rev. B, Condens. Matter, vol. 71, pp. 115326-1-115326-10, Mar. 2005.

[23] Z. Ikonic, R. W. Kelsall, and P. Harrison, "Waveguide design for mid- and far-infrared p-Si/SiGe quantum cascade lasers," Semicond. Sci. Technol., vol. 19, no. 1, pp. 76-81, 2004.
[24] M. Bain, H. A. W. El Mubarek, J. M. Bonar, Y. Yang, O. Buiu, H. Gamble B. M. Armstrong, P. L. F. Hemment, S. Hall, and P. Ashburn, "SiGe HBTs on bonded SOI incorporating buried silicide layers," IEEE Trans. Electron Devices, vol. 52, no. 3, pp. 317-324, Mar. 2005.

[25] V. N. Shastin, R. Kh. Zhukavin, E. E. Orlova, S. G. Pavlov, M. H. Rümmeli, H. W. Hübers, J. N. Hovenier, T. O. Klaassen, H. Riemann, I. V. Bradley, and A. F. G. van der Meer, "Stimulated terahertz emission from Group-V donors in silicon under intracenter photoexcitation," Appl. Phys. Lett., vol. 80, no. 19, p. 3512, May 2002.

[26] H.-W. Hübers, S. G. Pavlov, M. Greiner-Bär, M. H. Rümmeli, M. F Kimmit, R. Kh. Zhukavin, H. Riemann, and V. N. Shastin, "Terahertz emission spectra of optically pumped silicon lasers," Phys. Status Solidi, vol. 233, no. 2, pp. 191-196, 2002.

[27] S. G. Pavlov, H.-W. Hübers, M. H. Rümmeli, R. Kh. Zhukavin, E. E. Orlova, V. N. Shastin, and H. Riemann, "Far-infrared stimulated emission from optically excited bismuth donors in silicon," Appl. Phys. Lett., vol. 80, no. 25, pp. 4717-4719, Jun. 2002.

[28] H.-W. Hübers, S. G. Pavlov, H. Riemann, N. V. Abrosimov, R. Kh Zhukavin, and V. N. Shastin, "Stimulated terahertz emission from arsenic donors in silicon," Appl. Phys. Lett., vol. 84, no. 18, pp. 3600-3602, May 2004.

[29] S. G. Pavlov, H.-W. Hübers, H. Riemann, R. Kh. Zhukavin, E. E. Orlova, and V. N. Shastin, "Terahertz optically pumped Si:Sb laser," J. Appl. Phys., vol. 92, no. 10, pp. 5632-5634, Nov. 2002.

Stephen A. Lynch (S'98-A'99-M'01-SM'05) was born in Drogheda, Ireland, in 1973. He received the B.Sc. degree (with honors) in experimental physics from University College Dublin, Dublin, Ireland, in 1995, and the Ph.D. degree in physics from Trinity College Dublin, Dublin, in 2000.

He is currently with the Cavendish Laboratory, University of Cambridge, Cambridge, U.K. His current research interests include terahertz source and detector development, quantum cascade lasers, self-pulsating lasers, and secure telecommunications using chaotic encryption in pulsed laser diodes.

Douglas J. Paul (M'01-SM'05) was born in Greenock, U.K., in 1969. He received the B.A. and M.A. degrees in physics/theoretical physics in 1990 and 1994, respectively, and the Ph.D. degree, all from the University of Cambridge, Cambridge, U.K.

Since 1994, he has been with the Semiconductor Physics Group in the Cavendish Laboratory at the University of Cambridge. His current research interests include physics of short-channel CMOS devices, heterostructure and strained-Si complimentary metaloxidesemiconductor (CMOS), SiGe MODFETs, SiGe resonant tunneling diodes and quantum devices, $\mathrm{Si} / \mathrm{SiGe}$ quantum cascade lasers, terahertz technology, the physics of low-dimensional Si-based devices, and quantum information processing.

Paul Townsend (S'02), photograph and biography not available at the time of publication.

Guy Matmon (S'05), photograph and biography not available at the time of publication. 
Zhang Suet (S’02), photograph and biography not available at the time of publication.

Robert W. Kelsall, photograph and biography not available at the time of publication.

Zoran Ikonic was born in Belgrade, Yugoslavia, in 1956. He received the B.Sc., M.Sc., and Ph.D. degrees in electrical engineering from the University of Belgrade, Belgrade, Yugoslavia, in 1980, 1984, and 1987, respectively.

During 1981-1999, he was with the Faculty of Electrical Engineering, University of Belgrade, where he was a Full Professor from 1998. Since 1999, he has been with the School of Electronic and Electrical Engineering, University of Leeds, Leeds, U.K. His current research interests include electronic structure, optical and transport properties of semiconductor nanostructures, and devices based upon them.

Paul Harrison (M'99-SM'99) received the B.Sc. degree from the University of Hull, Hull, U.K., in 1988, and the Ph.D. degree from the University of Newcastle-upon-Tyne, Newcastle-upon-Tyne, U.K., in 1991.

He was a Postdoctoral Research Assistant at the University of Hull in 1995. He obtained a Fellowship at the University of Leeds, Leeds, U.K., and joined the Institute of Microwave and Photonics. He is currently Chair in quantum electronics and Head of the School of Electronic and Electrical Engineering, University of Leeds. He is the author of Quantum Wells, Wires and Dots (Wiley, 2005). He has been working on ways to adapt his theoretical and computational experience in semiconductor heterostructures to terahertz sources and detectors.

Jing Zhang, photograph and biography not available at the time of publication.

David J. Norris, photograph and biography not available at the time of publication.
Anthony G. Cullis, photograph and biography not available at the time of publication.

Carl R. Pidgeon, photograph and biography not available at the time of publication.

Pawel Murzyn, photograph and biography not available at the time of publication.

Ben Murdin, photograph and biography not available at the time of publication.

Mike Bain, photograph and biography not available at the time of publication.

Harry S. Gamble (M'96), photograph and biography not available at the time of publication.

Ming Zhao, photograph and biography not available at the time of publication.

Wei-Xin Ni, photograph and biography not available at the time of publication. 